Original article

Received: 22 November 2016 / Accepted: 17 January 2017

\title{
EVALUATING THE ACCURACY OF DETERMINING COORDINATES OF A CORNER OF A BUILDING MEASURED IN THE RTN GNSS MODE, HAVING APPLIED THE INNOVATIVE ALGORITHM OF VECTOR TRANSLATION
}

\author{
Robert Krzyżek \\ AGH University of Science and Technology, \\ Faculty of Mining Surveying and Environmental Engineering, \\ Department of Integrated Geodesy and Cartography
}

\begin{abstract}
The study evaluates the accuracy of determining coordinates of a corner of a building measured in the RTN GNSS mode (Real Time Network Global Navigation Satellite System) using the method of line-line intersection and having applied the algorithm of vector translation, developed by the author. The performed analysis of accuracy proved a high precision in determining the points subjected to studies. An important factor in the formation of a mean error regarding the position of the corner of a building, having used the algorithm of vector translation, is the assumption of correctness of the reference points, i.e. the so-called base points, determined in the RTN GNSS mode. In this case, the base points take the role of measurement control points. The mean error of the position of the corner of a building, taking into account the innovative solution, is at the level of several centimeters. The study results presented in the article allow to positively evaluate the algorithm of vector translation in terms of accuracy of determining the position of a corner of a building, measured in real time.
\end{abstract}

Keywords: RTN GNSS, vector translation, measurement of building structures, innovative algorithm

\section{Introduction}

Over the last twenty years, there has been significant progress in technological solutions used in the field of surveying. Surveying instruments used for measurements of field details are among such modern solutions. Modern equipment which provides numerous possibilities, contributes to the development of new, or improvement of the existing measurement technologies. One such technologies is the real time measurement RTN GNSS. Detailed analysis of the accuracy of this modern measuring method is widely known and used. However, there are continuous attempts to improve it in various research aspects, which would primarily result in the practical implementation of tasks in the field of surveying. In the case surveys performed in the 
RTN GNSS mode, the biggest challenge for researchers is to search for technological, design, and application solutions which will allow for the implementation of real-time measurements in difficult field conditions (e.g. the obscured horizon). The respective research was carried out by (Kyungho et al, 2009; Pelc-Mieczkowska, 2012; Bakuła, 2013; Dae et al, 2014). Also in (Krzyżek, 2015a, 2015c) original solutions developed by the author were proposed, largely contributing to the reliability of determining inaccessible field details for the RTN GNSS technology. All the solutions proposed by the aforementioned authors are closely related to other studies concerning, generally speaking, satellite measurements. They refer, for example, to the combined use of different satellite systems for the measurements in the RTN GNSS mode (Gunter, 2000; Pirti et al, 2013; Xingxing et al, 2015), to the development of reference station network in Poland (Wajda et al, 2008; Figurski et al, 2011), or to the application of various technical solutions for GNSS receivers.

Making use of the existing achievements of scientists in various research aspects of the RTN GNSS technology, the algorithm of vector translation, developed by the author, used to increase the reliability and accuracy of determining coordinates of a building structure measured in real time, was proposed in (Krzyżek, 2015a). The problem of reliability of the modified coordinates having applied this innovative algorithm, was examined in detail and presented in (Krzyżek, 2015b). However, this article concentrates mainly on the aspect of accuracy of determining coordinates of corners of a building structure, taking into account the method of vector translation.

The aim of the study presented in this article was to prove that observing the legal provisions which govern surveying in Poland, using the algorithm of vector translation for the measurement of building structures in the RTN GNSS mode will help to increase, or at least maintain, high accuracy of determining the position of their corners. For this purpose, a detailed mathematical analysis was conducted to prove how the mean error of the position of a building corner is formed and determined, when measured in the RTN GNSS mode, using the algorithm developed by the author.

\section{Mathematical analysis regarding the formation of the mean error of corner position after using the algorithm of vector translation}

For a more complete understanding of the analysis, Figure 1 illustrates essential components used in the method of vector translation. Due to the large volume of the study material, only one corner of the building - the point $d$ - for which all of the elements of the algorithm of vector translation are met (Krzyżek, 2015a), was used in the presented mathematical analysis. Accordingly, in order to fully illustrate the building geometry, Figure 2 presents the general outline of the structure with the base points $(A-L)$ measured in the RTN GNSS mode and with control measurement of tie distances $R$.

Moving directly to the mathematical analysis of the formation of the mean position error of a corner of a building, first of all, a formula to determine the coordinates $X, Y$ of the point $d$, having applied the method of vector translation, must be determined denoted as $X_{\widehat{d}}, Y_{\widehat{d}}$ : 


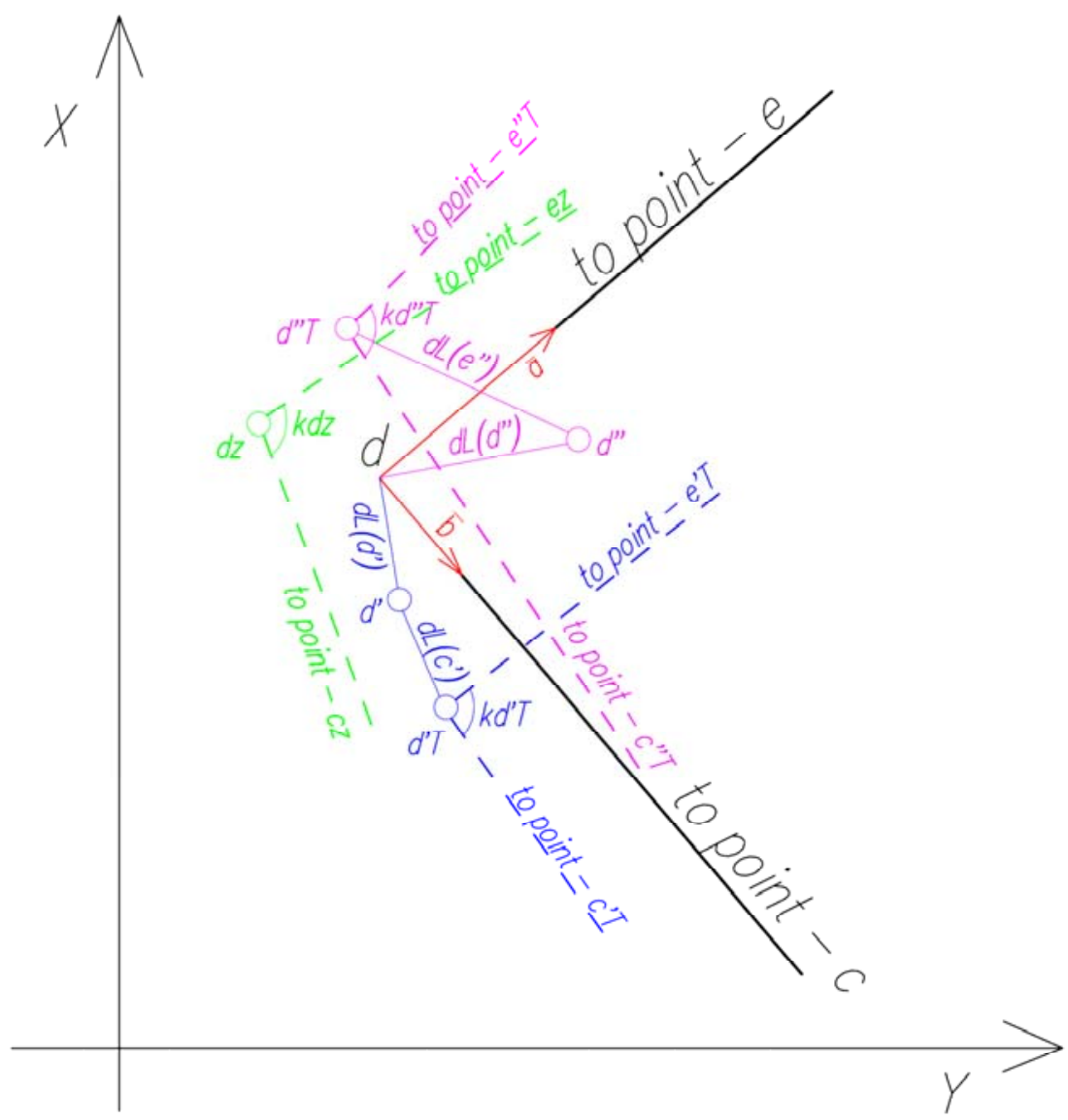

Fig. 1. Graphical presentation of the basic elements of the algorithm of vector translation.

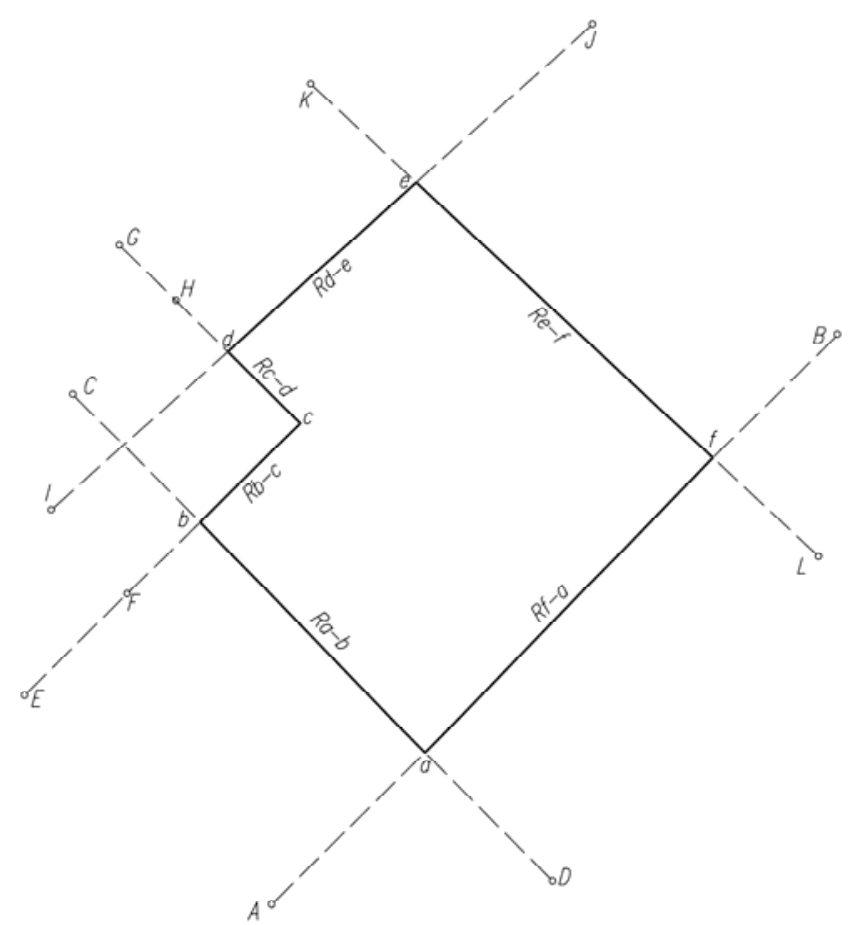

Fig. 2. Position of the base points $(A-L)$, measured in the RTN GNSS mode to determine corners of a building. 


$$
\begin{gathered}
X_{\hat{d}}=X_{\bar{a}}+X_{\bar{b}}-X_{d^{w}} \\
=\bar{a} \cdot \cos A_{d^{w}-c^{w}}+\bar{b} \cdot \cos A_{d^{w}-e^{w}}+\frac{X_{d_{T}} \cdot p^{\prime}{ }_{T}+X_{d^{\prime \prime}{ }_{T}} \cdot p^{\prime \prime}{ }_{T}+X_{d_{Z}} \cdot p_{Z}}{p_{T}^{\prime}+p^{\prime \prime}{ }_{T}+p_{Z}} \\
Y_{\widehat{d}}=Y_{\bar{a}}+Y_{\bar{b}}-Y_{d^{w}}=\bar{a} \cdot \sin A_{d^{w}-c^{w}}+\bar{b} \cdot \sin A_{d^{w}-e^{w}}+\frac{Y_{d_{T}} \cdot p_{T}^{\prime}+Y_{d^{\prime \prime}{ }_{T}} \cdot p^{\prime \prime}{ }_{T}+Y_{d_{Z}} \cdot p_{Z}}{p_{T}^{\prime}{ }_{T}+p^{\prime \prime}{ }_{T}+p_{Z}}
\end{gathered}
$$

where:

$X_{\hat{d}}, Y_{\hat{d}}$ - final coordinates of the corner of a building, taking into account the full algorithm of vector translation,

$X_{d^{w}}, Y_{d^{w}}$ - coordinates the corner of a building, determined as a weighted average (excluding the last component - the method of vector addition - the algorithm of vector translation),

$X_{\bar{a}}, X_{\bar{b}}, Y_{\bar{a}}, Y_{\bar{b}}$, - coordinates of the terminal points of the vectors $\bar{a}$ and $\bar{b}$ used in the method of vector addition,

$A_{d^{w}-c^{w}}, A_{d^{w}-e^{w}}$ - azimuths of the respective wall faces in a building,

$X_{d_{T}{ }_{T}}, X_{d^{\prime \prime} T_{T}}, X_{d_{Z}}, Y_{d^{\prime} T}, Y_{d^{\prime \prime} T_{T}}, Y_{d_{Z}}$ - coordinates of the corner of a building, determined in the main direction $\left(X_{d^{\prime} T}, Y_{d^{\prime} T}\right)$, counter direction $\left(X_{d^{\prime \prime} T}, Y_{d^{\prime \prime} T}\right)$, and from the line-line intersection $\left(X_{d_{Z}}, Y_{d_{Z}}\right)$ of the wall faces in a building, according to the algorithm of vector translation (Krzyżek, 2015a),

$p^{\prime}{ }_{T}, p^{\prime \prime}{ }_{T}, p_{Z}$ - the weights calculated according to the algorithm of vector translation (Krzyżek, 2015a).

In order to specify the mean errorm $m_{\hat{d}}$ of the point which is being determined, the law of propagation of errors should be applied to the equation (1):

$$
m_{P_{\widehat{d}}}=\sqrt{m_{\bar{a}}^{2}+m_{\bar{b}}^{2}+a^{2} \cdot m_{A_{d^{w}-c^{w}}}^{2}+b^{2} \cdot m_{A_{d^{w}-e^{w}}}^{2}+m_{X_{d^{w}}}^{2}+m_{Y_{d^{w}}}^{2}}
$$

Further considerations focus on a very important factor, which is the assumption of correctness of the base points $(A-L)$ - Fig. 2 - determined in the RTN GNSS mode, which can be defined as measurement control points.

In accordance with the general knowledge and evaluation of the accuracy of the field details being measured, as contained in (MIA, 2011), reference points (in this case of the measurement control), are assumed to be error-free. According to this assumption, all data (azimuths, angles, etc.), calculated based on these coordinates, are also assumed to be error-free, which can be described by the general equation:

$$
m_{N}=m_{a-f}=m_{A / v}=m_{k}=0
$$

where:

$m_{N}$ - mean errors of the position of the base points $(A-L)$ determined in the RTN GNSS mode - the assumption which was adopted and substantiated in (Krzyżek, 2014), $m_{a-f}$ - mean errors of the position of the points (corners of the building a-f) determined from the line-line intersection of the appropriate base points $(A-L)$, $m_{A / v}$ - mean error of the azimuth $A$ (or the angular correction $v$ ) of the respective wall face in a building,

$m_{k}$ - mean error of the angle $k$. 
In order to solve the equation (2), first of all, the values of the vectors $\bar{a}$ and $\bar{b}$ must be determined, and then mean errors of individual components of the formula (2).

According to the algorithm of vector addition (developed and examined in detail by the author), the vectors $\bar{a}$ and $\bar{b}$ relative to the wall faces of the building (for the corner d), are calculated as:

$$
\begin{aligned}
& \bar{a}=\frac{1}{2}\left(R_{d-c}^{w}-R_{d-c}^{p}\right) \\
& \bar{b}=\frac{1}{2}\left(R_{d-e}^{w}-R_{d-e}^{p}\right)
\end{aligned}
$$

where:

$R_{d-c}^{w}, R_{d-e}^{w}$ - the lengths of the wall faces of the building ( $d-c$ and $\left.d-e\right)$, determined based on the coordinates,

$R_{d-c}^{p}, R_{d-e}^{p}$ - the lengths of the wall faces of the building ( $d-c$ and $\left.d-e\right)$ determined based on the control measurement (of tie distances).

Using the Gauss' law for the equation (4), while taking into account the equation (3), the mean errors of the vectors $\bar{a}$ and $\bar{b}$ can be determined:

$$
\begin{aligned}
& m_{\bar{a}}=\frac{1}{2} m_{R_{d-c}^{p}} \\
& m_{\bar{b}}=\frac{1}{2} m_{R_{d-e}^{p}}
\end{aligned}
$$

The next step is calculating the mean errors of the azimuths $m_{A_{d^{w}-c^{w}}}, m_{A_{d^{w}-e^{w}}}$ of the wall faces of the building. Therefore, the coordinates $X, Y$ of the corners $c, d, e$, must be determined according to the algorithm of vector translation (Krzyżek, 2015a) as weighted averages:

- for the point $d$ :

- for the point c:

$$
\begin{aligned}
& X_{d^{w}}=\frac{X_{d^{\prime} T} \cdot p_{d^{\prime}{ }_{T}}+X_{d^{\prime \prime}{ }_{T}} \cdot p_{d^{\prime \prime}{ }_{T}}+X_{d_{Z}} \cdot p_{d_{Z}}}{p_{d^{\prime}{ }_{T}}+p_{d^{\prime \prime}{ }_{T}}+p_{d_{Z}}}
\end{aligned}
$$

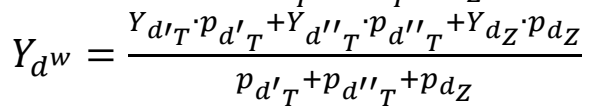

$$
\begin{aligned}
& X_{C^{w}}=\frac{X_{C^{\prime} T} \cdot p_{c^{\prime} T_{T}}+X_{c^{\prime \prime} T_{T}} \cdot p_{c^{\prime \prime} T_{T}}+X_{C_{Z}} \cdot p_{c_{Z}}}{p_{c^{\prime} T}+p_{c^{\prime \prime}{ }_{T}}+p_{c_{Z}}}
\end{aligned}
$$

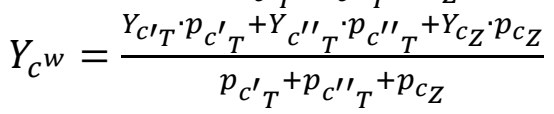

- for the point e:

$$
\begin{aligned}
X_{e^{w}} & =\frac{X_{e^{\prime} T} \cdot p_{e^{\prime} T_{T}}+X_{e^{\prime \prime}{ }_{T} \cdot p_{e^{\prime \prime}}+X_{e_{Z}} \cdot p_{e_{Z}}}}{p_{e^{\prime} T}+p_{e^{\prime \prime} T}+p_{e_{Z}}} \\
Y_{e^{w}} & =\frac{Y_{e^{\prime} T_{T}} \cdot p_{e^{\prime} T_{T}}+Y_{e^{\prime \prime} T} \cdot p_{e^{\prime \prime} T_{T}}+Y_{e_{Z}} \cdot p_{e_{Z}}}{p_{e^{\prime}{ }_{T}}+p_{e^{\prime \prime}{ }_{T}}+p_{e_{Z}}}
\end{aligned}
$$

The components of the formulas (6) - (11) have been presented in the legend to the equation (1). 
Using the equations (6) - (11), azimuths of the sides $A_{d^{w}-c^{w}}, A_{d^{w}-e^{w}}$ were calculated, and then the mean errors $m_{A_{d^{w}{ }_{-} c^{w}}}, m_{A_{d^{w} e_{-} w^{w}}}$ of the determined azimuths:

where:

$$
\begin{aligned}
& m_{A_{d^{w}-c^{w}}}=\sqrt{\left(s_{1}+q_{1}\right)} \cdot m_{N} \\
& m_{A_{d^{w}-e^{w}}}=\sqrt{\left(s_{2}+q_{2}\right)} \cdot m_{N}
\end{aligned}
$$

$$
\begin{aligned}
& s_{1}=\left(\frac{1}{r_{1}+1}+\frac{1}{X_{c^{w}}-X_{d^{w}}}\right)^{2}+\left(\frac{1}{r_{1}+1}-\frac{1}{X_{c^{w}}-X_{d^{w}}}\right)^{2} \\
& q_{1}=\left(\frac{1}{r_{1}+1}+\frac{Y_{d^{w}}-Y_{c^{w}}}{\left(X_{c^{w}}-X_{\left.d^{w}\right)^{2}}\right.}\right)^{2}+\left(\frac{1}{r_{1}+1}-\frac{Y_{c^{w}}-Y_{d^{w}}}{\left(X_{c^{w}}-X_{d^{w}}\right)^{2}}\right)^{2} \\
& s_{2}=\left(\frac{1}{r_{2}+1}+\frac{1}{X_{e^{w}}-X_{d^{w}}}\right)^{2}+\left(\frac{1}{r_{2}+1}-\frac{1}{X_{e^{w}}-X_{d^{w}}}\right)^{2} \\
& q_{2}=\left(\frac{1}{r_{2}+1}+\frac{Y_{d^{w}}-Y_{e^{w}}}{\left(X_{e^{w}}-X_{d^{w}}\right)^{2}}\right)^{2}+\left(\frac{1}{r_{2}+1}-\frac{Y_{e^{w}}-Y_{d^{w}}}{\left(X_{e^{w}}-X_{d^{w}}\right)^{2}}\right)^{2} \\
& r_{1}=\left(\frac{Y_{c^{w}}-Y_{d^{w}}}{X_{c^{w}}-X_{d^{w}}}\right)^{2} \\
& r_{2}=\left(\frac{Y_{e^{w}}-Y_{d^{w}}}{X_{e^{w}}-X_{d^{w}}}\right)^{2}
\end{aligned}
$$

The last step to solve the equation (2) is to determine the mean errors $m_{X_{d^{w}}}, m_{Y_{d^{w}}}$ of the corner of the building denoted as $d^{w}$.Thus, for the equations (6) and (7), partial derivatives are calculated, while introducing additional denotations:

$$
\begin{aligned}
& \left(\frac{\partial X_{d^{w}}}{\partial X_{d^{\prime} T}}\right)^{\prime}=\left(\frac{\partial Y_{d^{w}}}{\partial Y_{d^{\prime} T}}\right)^{\prime}=\frac{p_{d^{\prime} T}}{p_{d^{\prime} T}+p_{d^{\prime \prime}{ }_{T}}+p_{d_{Z}}}=A_{X}=A_{Y} \\
& \left(\frac{\partial X_{d^{w}}}{\partial X_{d{ }^{\prime \prime} T}}\right)^{\prime}=\left(\frac{\partial Y_{d^{w}}}{\partial Y_{d{ }^{\prime \prime} T}}\right)^{\prime}=\frac{p_{d{ }^{\prime \prime} T}}{p_{d^{\prime} T}+p_{d^{\prime \prime} T_{T}}+p_{d_{Z}}}=B_{X}=B_{Y} \\
& \left(\frac{\partial X_{d^{w}}}{\partial X_{d_{Z}}}\right)^{\prime}=\left(\frac{\partial Y_{d^{w}}}{\partial Y_{d_{Z}}}\right)^{\prime}=\frac{p_{d_{Z}}}{p_{d^{\prime} T}+p_{d^{\prime \prime} T}+p_{d_{Z}}}=C_{X}=C_{Y}
\end{aligned}
$$

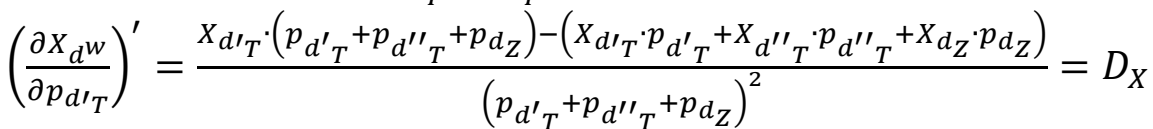

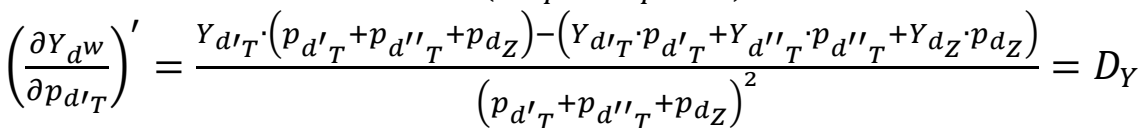

$$
\begin{aligned}
& \left(\frac{\partial X_{d^{w}}}{\partial p_{d^{\prime \prime} T}}\right)^{\prime}=\frac{X_{d^{\prime \prime} T} \cdot\left(p_{d^{\prime} T_{T}}+p_{d^{\prime \prime}{ }_{T}}+p_{d_{Z}}\right)-\left(X_{d^{\prime} T_{T}} \cdot p_{d^{\prime} T_{T}}+X_{d^{\prime \prime} T_{T}} \cdot p_{d^{\prime \prime} T_{T}}+X_{d_{Z}} \cdot p_{d_{Z}}\right)}{\left(p_{d^{\prime} T}+p_{d^{\prime \prime} T_{T}}+p_{d_{Z}}\right)^{2}}=E_{X}
\end{aligned}
$$

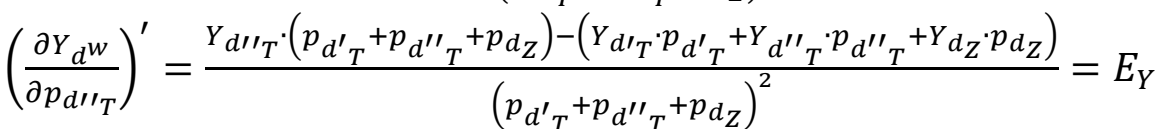

$$
\begin{aligned}
& \left(\frac{\partial X_{d^{w}}}{\partial p_{d_{Z}}}\right)^{\prime}=\frac{X_{d_{Z}} \cdot\left(p_{d^{\prime}{ }_{T}}+p_{d^{\prime \prime} T_{T}}+p_{d_{Z}}\right)-\left(X_{d^{\prime} T_{T}} \cdot p_{d^{\prime} T_{T}}+X_{\left.d^{\prime \prime} T_{T} \cdot p_{d^{\prime \prime}{ }_{T}}+X_{d_{Z}} \cdot p_{d_{Z}}\right)}\right.}{\left(p_{d^{\prime}{ }_{T}}+p_{d^{\prime \prime}{ }_{T}}+p_{d_{Z}}\right)^{2}}=F_{X}
\end{aligned}
$$

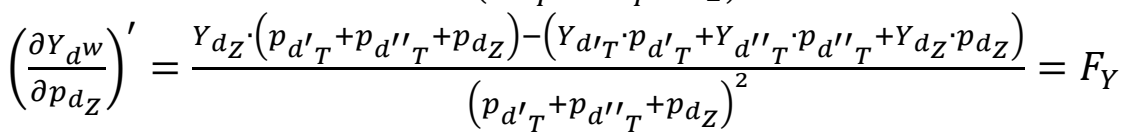


and (having applied the law of propagation of errors) the following is obtained:

$$
\begin{aligned}
m_{X_{d^{w}}} & =\sqrt{A_{X}^{2} \cdot m_{X_{d^{\prime} T}}^{2}+B_{X}^{2} \cdot m_{X_{d^{\prime \prime} T}}^{2}+C_{X}^{2} \cdot m_{X_{d_{Z}}}^{2}+D_{X}^{2} \cdot m_{{p_{d^{\prime} T}}^{\prime}}^{2}+E_{X}^{2} \cdot m_{p_{d^{\prime \prime} T}}^{2}+F_{X}^{2} \cdot m_{p_{d_{Z}}}^{2}}(23) \\
m_{Y_{d^{w}}} & =\sqrt{A_{Y}^{2} \cdot m_{Y_{d^{\prime} T}}^{2}+B_{Y}^{2} \cdot m_{Y_{d^{\prime \prime} T}}^{2}+C_{Y}^{2} \cdot m_{Y_{d_{Z}}}^{2}+D_{Y}^{2} \cdot m_{{p_{d^{\prime}}}^{\prime}}^{2}+E_{Y}^{2} \cdot m_{{p_{d^{\prime \prime}}}_{T}}^{2}+F_{Y}^{2} \cdot m_{{p_{d_{Z}}}}^{2}}(24)
\end{aligned}
$$

To be able to carry out the final assessment of the accuracy of the position of the corner $d$, having applied the algorithm of vector translation, the formation of mean errors contained in the equations (23) and (24) should be determined. The mean errors $m_{X_{d{ }^{\prime} T}}, m_{Y_{d^{\prime} T}}, m_{X_{d \prime \prime}{ }^{\prime}}, m_{Y_{d^{\prime \prime} T}}$ are dependent upon the mean position errors of the corner points of the building determined in the main direction $m_{X_{d \prime}}, m_{Y_{d}} m_{X_{C^{\prime}}}, m_{Y_{C^{\prime}}}$ and in the counter direction $m_{X_{d \prime \prime}}, m_{Y_{d \prime \prime}} m_{X_{e \prime \prime}}, m_{Y_{e \prime}}$, having applied the method of vector translation (Krzyżek, 2015a). Taking into account equation (3) in these dependencies, it is:

$$
\begin{aligned}
& m_{X_{d^{\prime} T}}=\sqrt{m_{X_{d \prime}}^{2}+m_{X_{c^{\prime}}}^{2}} \\
& m_{Y_{d^{\prime} T}}=\sqrt{m_{Y_{d \prime}}^{2}+m_{Y_{C \prime}}^{2}} \\
& m_{X_{d{ }^{\prime \prime} T}}^{2}=\sqrt{m_{X_{d \prime \prime}}^{2}+m_{X_{e \prime \prime}}^{2}} \\
& m_{Y_{d^{\prime \prime} T}}=\sqrt{m_{Y_{d \prime \prime}}^{2}+m_{Y_{e^{\prime \prime}}}^{2}}
\end{aligned}
$$

The individual mean errors of the corners of the building, $c, d$, e, determined in the main direction $c^{\prime}, d^{\prime}$, and in the counter direction $e^{\prime \prime} d^{\prime \prime}$ (Krzyżek, 2015a), contained in the equations (25) - (28), according to the equation (3) are as follows:

$$
\begin{aligned}
& m_{X_{d \prime}}=\sqrt{\cos ^{2}\left(A_{c-d}+v_{k_{c}}\right) \cdot m_{R_{c-d}^{p}}^{2}} \\
& m_{Y_{d \prime}}=\sqrt{\sin ^{2}\left(A_{c-d}+v_{k_{c}}\right) \cdot m_{R_{c-d}}^{2}} \\
& m_{X_{c^{\prime}}}=\sqrt{\cos ^{2}\left(A_{b-c}+v_{k_{b}}\right) \cdot m_{R_{b-c}^{p}}^{2}} \\
& m_{Y_{c^{\prime}}}=\sqrt{\sin ^{2}\left(A_{b-c}+v_{k_{b}}\right) \cdot m_{R_{b-c}^{p}}^{2}} \\
& m_{X_{d \prime \prime}}=\sqrt{\cos ^{2}\left(A_{e-d}+v_{k_{e}}\right) \cdot m_{R_{e-d}}^{2}} \\
& m_{Y_{d \prime \prime}}=\sqrt{\sin ^{2}\left(A_{e-d}+v_{k_{e}}\right) \cdot m_{R_{e-d}^{p}}^{2}} \\
& m_{X_{e \prime \prime}}=\sqrt{\cos ^{2}\left(A_{f-e}+v_{k_{f}}\right) \cdot m_{R_{f-e}^{p}}^{2}} \\
& m_{Y_{e \prime}}=\sqrt{\sin ^{2}\left(A_{f-e}+v_{k_{f}}\right) \cdot m_{R_{f-e}^{p}}^{2}}
\end{aligned}
$$


On the other hand, the mean errors $m_{X_{d_{Z}}}, m_{Y_{d_{Z}}}$ - the equations (23) and (24) - result from the Gaussian model used for the coordinates $X_{d_{Z}}, Y_{d_{Z}}$ calculated (according to the algorithm of vector translation) from the intersection of the lines: the base line $d^{\prime}{ }_{T}-c^{\prime}{ }_{T}$ and the cutting line $e^{\prime \prime}{ }_{T}-d^{\prime \prime}{ }_{T}$ :

where:

$$
\begin{aligned}
& X_{d_{Z}}=\frac{Y_{d^{\prime} T}-Y_{d^{\prime \prime} T}+\lambda \cdot X_{d^{\prime \prime} T}-\mu \cdot X_{d^{\prime} T}}{\lambda-\mu} \\
& Y_{d_{Z}}=Y_{d^{\prime \prime} T}+\lambda\left(X_{d_{Z}}-X_{d^{\prime \prime} T}\right)
\end{aligned}
$$

$\lambda=\operatorname{tg} A_{d \prime \prime T}-e \prime^{\prime \prime}$

$\mu=\operatorname{tg} A_{d^{\prime} T-C^{\prime} T}$

and they are as follows:

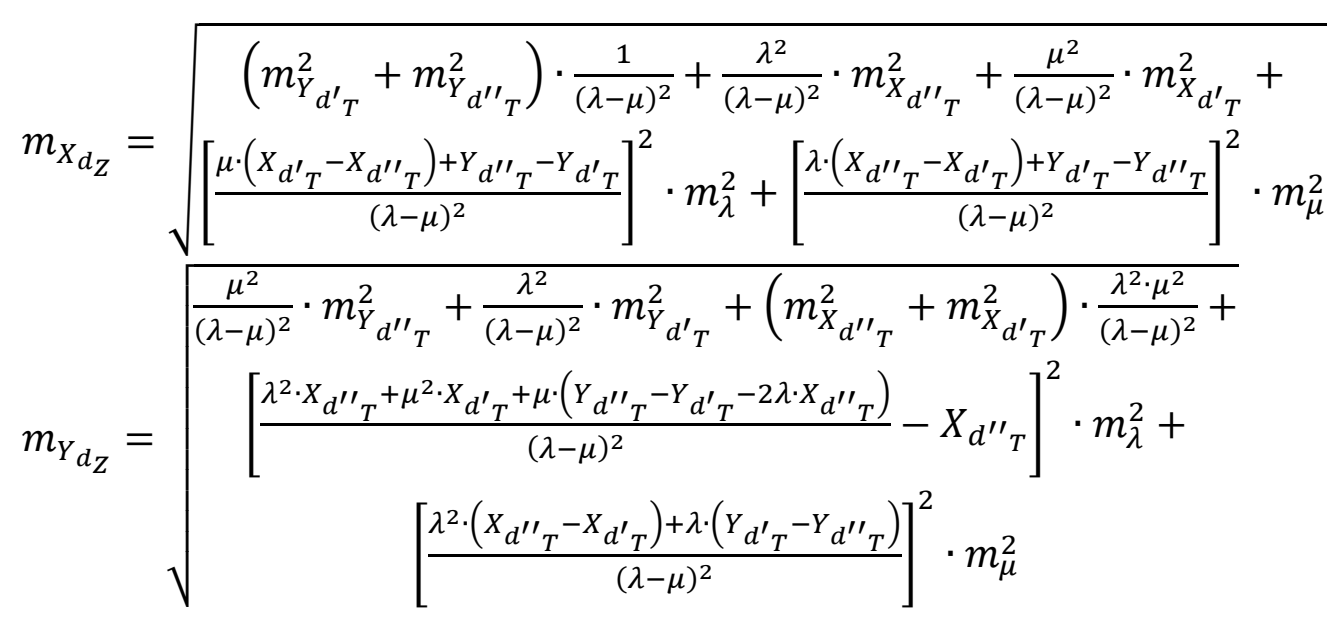

One of the most important factors of the algorithm of vector translation are the weights $p_{d^{\prime}{ }_{T}}, p_{d^{\prime \prime}{ }^{\prime},}, p_{d_{Z}}$ of the corner $d$, which are inversely proportional to the square of the angular correction, respectively $v_{k_{d \prime_{T}}}, v_{k_{d \prime^{\prime} T}}, v_{k_{d_{Z}}}$ :

where:

$$
\begin{aligned}
& p_{d{ }^{\prime} T}=\frac{1}{v_{k_{d \prime}{ }^{2}}{ }^{2}} \\
& p_{d \prime^{\prime} T}=\frac{1}{v_{k_{d \prime^{\prime \prime} T}}{ }^{2}} \\
& p_{d_{z}}=\frac{1}{v_{k_{d_{Z}}}{ }^{2}}
\end{aligned}
$$

$v_{k_{d^{\prime} T}}=\frac{90^{0}-k_{d^{\prime} T}}{2}$

$v_{k_{d \prime \prime} T}=\frac{90^{0}-k_{d \prime^{\prime} T}}{2}$

$v_{k_{d_{Z}}}=\frac{90^{0}-k_{d_{Z}}}{2}$

$k_{d^{\prime} T}, k_{d^{\prime \prime} T}, k_{d_{Z}}-$ horizontal angles of the corner $d$, determined according to the algorithm of vector translation (Krzyżek, 2015a). 
Errors of the weights $m_{p_{d^{\prime} T}}, m_{p_{d^{\prime \prime} T},} m_{p_{d_{Z}}}$ - for the equations (23) and (24) - are determined using once again the law of propagation of errors to the equations (41) (43):

$$
\begin{aligned}
& m_{p_{d^{\prime} T}}=\sqrt{\left(\frac{8}{90^{0}-k_{d^{\prime} T}}\right)^{2} \cdot m_{k_{d^{\prime} T}}^{2}} \\
& m_{p_{d^{\prime \prime} T}}=\sqrt{\left(\frac{8}{90^{0}-k_{d \prime^{\prime} T}}\right)^{2} \cdot m_{k_{d^{\prime \prime} T}}^{2}} \\
& m_{p_{d_{Z}}}=\sqrt{\left(\frac{8}{90^{0}-k_{d_{Z}}}\right)^{2} \cdot m_{k_{d_{Z}}}^{2}}
\end{aligned}
$$

where:

$m_{k_{d^{\prime} T^{\prime}}}, m_{k_{d^{\prime \prime} T^{\prime}}}, m_{k_{d_{Z}}}$-mean errors of the horizontal angles $k_{d^{\prime} T}, k_{d^{\prime \prime} T}, k_{d_{Z}}$ (calculated from the differences in the respective azimuths) of the corner $d$, determined basing on the Gaussian formula:

$$
\begin{aligned}
& m_{k_{d^{\prime} T}}=\sqrt{m_{A_{d^{\prime}-c c^{\prime}}}^{2}+m_{A_{d^{\prime}-e \prime}}^{2}} \\
& m_{k_{d^{\prime \prime} T}}=\sqrt{m_{A_{d^{\prime \prime}-c \prime \prime}}^{2}+m_{A_{d^{\prime \prime}-e \prime^{\prime \prime}}}^{2}} \\
& m_{k_{d_{Z}}}=\sqrt{m_{A_{d_{Z}-c_{Z}}}^{2}+m_{A_{d_{Z}-e_{Z}}}^{2}}
\end{aligned}
$$

\section{Evaluating the accuracy of measurement results, having applied the algorithm of vector translation}

The equations presented above allow to evaluate the accuracy of the method of vector translation, which will substantiate high precision of the modified coordinates of the corners of the building determined in the RTN GNSS mode. Given the assumption of the equation (3) and the mean errors of the linear measurement (with open frame tape measure) $m_{R_{d-c}^{p}}=m_{R_{d-e}^{p}}$ from the equation (5) at the level of individual centimeters, the final mean errors of the position of the corner of the building - the equation (2) have been presented in Table 1.

Tab. 1.Mean errors of the position of the corner of the building, having applied the algorithm of vector translation

\begin{tabular}{|c|c|}
\hline $\boldsymbol{m}_{\boldsymbol{R}_{\boldsymbol{d}-\boldsymbol{c}}^{\boldsymbol{p}}}=\boldsymbol{m}_{\boldsymbol{R}_{\boldsymbol{d}-\boldsymbol{e}}^{\boldsymbol{p}}}[\mathrm{m}]$ & $\boldsymbol{m}_{\boldsymbol{P}_{\mathfrak{d}}}[\mathrm{m}]$ \\
\hline 0.01 & 0.015 \\
\hline 0.02 & 0.027 \\
\hline 0.03 & 0.040 \\
\hline
\end{tabular}

A graphical presentation of the results contained in Table 1 have been illustrated in Figure 3. 


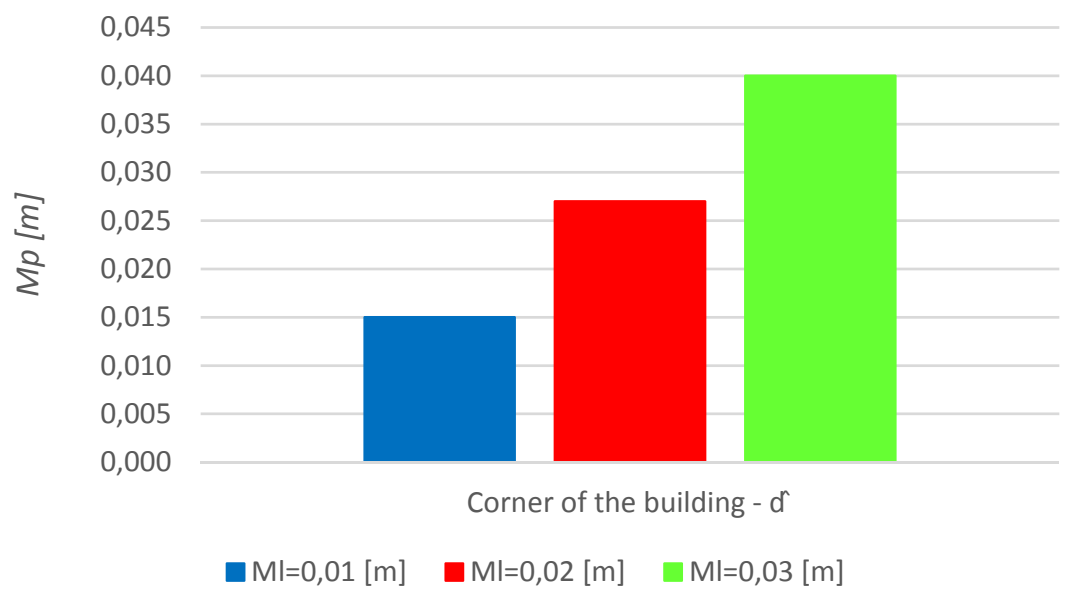

Fig.3. Mean errors of the position of the corner of the building, depending on the error of linear measurement, having applied the algorithm of vector translation.

When referring to the practical assessment of the accuracy of determining corners of buildings measured in the RTN GNSS mode using the algorithm of vector translation, one of the important aspects of the research should strongly emphasized. It mainly concerns the assumptions presented in the equation (3). The equation (3) is focused primarily on the correctness of the base points determined in real time. Such an assumption entails the adoption of all the elements calculated based on the coordinates of the base points as error-free. Such a course of calculations is in line with (MIA, 2011). Obviously, one should be aware that any observation performed in the field is always subject to a measurement error.

However, having used the method of vector translation, the assumptions adopted from the equation (3) lead to the formation of mean errors of the corner of the building (Tab. 1, Fig. 3) at a very high level of accuracy. This allows for a statement that the implementation of the innovative solution will provide not only the reliability of the results (Krzyżek, 2015b), but also a low level of mean errors of the obtained results.

\section{Summary}

In assessing the accuracy of determining corners of a building measured in the RTN GNSS mode, one should be fully aware of all the factors involved in the formation of the final value of the mean position error of the measured field detail. The process of accuracy analysis can be carried out in two different directions. The first one involves adopting all the observations and calculation results with specific errors. According to the law of propagation of errors, for further calculations in the accuracy analysis, mean errors of the control on which the whole measurement was based, should be assumed. The provisions contained in (MIA, 2011) allow the mean position error of the measurement control (the base points, in this case) at the level of $\pm 0.10 \mathrm{~m}$. The same applies to the maximum mean position error of a field detail, which is the corner of a building. In the case of large (but acceptable) position errors of the control, it could turn out that the accuracy of determining the position of a corner of a building exceeds $\pm 0.10 \mathrm{~m}$ (regardless of the method of measurement). Performance of the accuracy analysis in this direction is a theoretical consideration. 
This article focuses primarily on the possibilities of practical applications of innovative solutions (the algorithm of vector translation) in the field of surveying and cartography. Therefore, the second line of research has been selected in the process of accuracy analysis, which is assumption of correctness of the parameters presented in the equation (3). They are mainly based on the assumption of correctness of the reference points (base points). Such a course of conduct is adopted for the implementation of the accuracy analysis of the field details determined with detailed measurements, which include, for example, RTN GNSS technology. The research results presented in the article allow for a positive evaluation of the algorithm of vector translation in terms of the accuracy of determining the position of a corner of a building. Assuming linear measurement errors (with open frame tape measure) at the level of $\pm 0.01 \mathrm{~m}, \pm 0.02 \mathrm{~m}$, or even $\pm 0.03 \mathrm{~m}$, it is noticeable that the accuracy of the position of the corner of a building (mean error) does not exceed even half the value of the allowable position error of the field detail.

In conclusion, it must be assumed that the algorithm of vector translation not only increases the reliability of the determined coordinates of a corner of a building (Krzyżek, 2015b), but it also allows to maintain high precision of its determination, and therefore the mean errorm $P_{\widehat{d}}$ of the point position remains at a low level (from $0.015 \mathrm{~m}$ to $0.040 \mathrm{~m}$ ).

\section{Acknowledgement}

This work was carried out within the statutory studies of the AGH University of Science and Technology, Faculty of Mining Surveying and Environmental Engineering, Department of Integrated Geodesy and Cartography No. 11.11.150.444.

\section{References}

Bakuła, M. (2013). Study of Reliable Rapid and Ultrarapid Static GNSS Surveying for Determination of the Coordinates of Control Points in Obstructed Conditions. Journal of Surveying Engineering,139(4), pp. 188-193. DOI: 10.1061/(ASCE)SU.1943-5428.0000109

Dae Hee, W., Eunsung, L., Moonbeom, H., Sangkyung, S., Jiyun L., \& Young Jae L. (2014). GNSS integration with vision-based navigation for low GNSS visibility conditions. GPS Solution, 18(2), pp. 177-187. DOI: 10.1007/s10291-013-0318-8

Figurski, M., Bogusz, J., Bosy, J., Kontny, B., Krankowski, A., \& Wielgosz, P. (2011). ASG+: project for improving Polish multifunctional precise satellite positioning system. Reports on Geodesy, 2(91), pp. 51-57

Gunter W, Hein. (2000). From GPS and GLONASS via EGNOS to Galileo Positioning and Navigation in the Third Millennium. GPS Solutions, 3(4), pp. 3947. DOI: 10.1007/PL00012814

Krzyżek, R. (2014a). Reliability analysis of the results of RTN GNSS surveys of building structures using indirect methods of measurement. Geodesy and Cartography, 63(2), pp. 161-181. DOI: 10.2478/geocart-2014-0012

Krzyżek, R. (2015a). Algorithm for modeling coordinates of corners of buildings determined with RTN GNSS technology using vector translation method. Artificial Satellites Journal of Planetary Geodesy, 50(3), pp. 115-125. DOI: 10.1515/arsa2015-0009

Krzyżek, R. (2015b). Mathematical analysis of the algorithms used in modernized methods of building measurements with RTN GNSS technology. Boletim de Ciências Geodésicas, 21(4), pp. 848-866 
Krzyżek, R.(2015c). Modernization of the method of line-line intersection using RTN GNSS technology for determining the position of corners of buildings. Artificial Satellites Journal of Planetary Geodesy, 50(1), pp. 41-57.DOI: 10.1515/arsa-20150004

Kyungho, Y., Sangkyung, S., Eunsung, L., Sanguk, L., Jaehoon, K., Ho-Jin, L., \& Young Jae L. (2009). Availability assessment of GPS augmentation system using geostationary satellite and QZSS in Seoul Urban Area. Transactions of the Japan Society for Aeronautical and Space Sciences, 52(177), pp. 152-158. doi.org./102322/tjsass.52.152

MIA.(2011). Regulation of Minister of Interior and Administration - in case of technical standards of performing detailed surveys and working out and sending results of these surveys to National Geodetic and Cartographic Database (in Polish). Journal of Laws 263 (entry 1572), Warsaw: Government Legislation Centre.

Pelc-Mieczkowska, R. (2012). Analysis of GPS/RTK positioning reliability in hard observational conditions. Zeszyty Naukowe Politechniki Rzeszowskiej, 59(1/II), pp. 217-226

Pirti, A., Yucel, M.,\& Gumus, K. (2013). Testing Real Time Kinematics GNSS (GPS and GPS/GLONASS) methods in obstructed and unobstructed sites. Geodetski Vestnik, 57(3), pp. 498-512. DOI: 10.15292/geodetski-vestnik.2013.03.498-512.

Wajda, S., Oruba, A.,\& Leończyk, M. (2008). Technical details of establishing reference station network ASG-EUPOS. Paper presented at the Geoinformation Challenges, GIS Polonia 2008 Conference Proceedings, University of Silesia, Sosnowiec.

Xingxing, L., Maorong, G., Xiaolei, D., Xiaodong, R., Mathias, F., Jens, W., \& Harald, S. (2015). Accuracy and reliability of multi-GNSS real-time precise positioning: GPS, GLONASS, BeiDou, and Galileo. Journal of Geodesy, 89(6), pp. 607-635. DOI: 10.1007/s00190-015-0802-8.

\section{Author:}

Robert Krzyżek 1), rkrzyzek@agh.edu.pl

1) AGH University of Science and Technology,

Faculty of Mining Surveying and Environmental Engineering,

Department of Integrated Geodesy and Cartography

30 Mickiewicza Av., 30-059 Krakow, Poland 